\title{
The way you say it
}

\author{
Wording criticism constructively is important before and after publication.
}

German speakers put it well: 'Der Ton macht die Musik'it is the tone that makes the music. In other words, how something is said is as important as the content of the message. Scientific discourse is no exception, and harsh or offensive words can easily obscure valid criticism.

One good illustration is the controversy around the interpretation of Encyclopedia of DNA elements (ENCODE) data. September 2012 saw the publication of over 30 papers describing the enormous resources the ENCODE Consortium had assembled together with its conclusion that $80 \%$ of the human genome is functional. This triggered extensive and sometimes heated criticism from several researchers who strongly disagreed with this conclusion. One critique in particular (D. Graur et al., Genome Biol. Evol. 5, 578-590, 2013) pulled no punches in condemning ENCODE's interpretation of their data.

In a talk at the Society for Molecular Biology and Evolution in July, the paper's corresponding author, Dan Graur, shared that one high-impact genetics journal had rejected his work but recommended a "reasoned and dispassionate critical essay on the topic" instead.

Would a dispassionate and polite reply have been less visible? Is provocation necessary to get attention from a 'big science' consortium such as ENCODE? We do not think so. W. Ford Doolittle (Proc. Natl. Acad. Sci. USA 110, 5294-5300, 2013) and Sean R. Eddy (Curr. Biol. 23, R259-R261, 2013) provide good examples for respectful yet decisive disagreement with ENCODE's conclusions.

At Nature Methods, some of our published papers have elicited criticism; in our experience, respectful communication is key to a productive dialogue that advances science. There are several ways to bring one's disagreement with conclusions of a paper to the attention of the editors and the authors. The best first step is to write a letter to the editor outlining the problem and proposing a solution. If the criticism is substantial, the original authors are then given the chance to reply, and the exchange is sent for peer review. Over the years we have published several such exchanges. None of the critics saw the need to insult the original authors: instead they highlighted, for example, disagreement about the interpretation of data and areas that need more methods development (R.M. Jonker et al., Nat. Methods 10, 373, 2013; and other Correspondence from the same issue).

Another possibility for a researcher dissatisfied with the work of others is to build on and expand, rather than disparage, it: on p. 926 of this issue, Göttgens, Ruau and colleagues present HAEMCODE, a database of binding data for 77 transcription factors in 22 blood cell types- curated from published, mostly small-scale papersadding information to ENCODE, which profiled fewer transcription factors in hematopoietic cell lines. With this resource, the authors make their point that 'small science, when efficiently collated, can provide resources on the scale of big science efforts.

Civility in discourse is also essential before publication. We often receive strongly worded replies in the course of the peer review process. Receiving a rejection of one's own work can understandably trigger strong emotions. We are not saying that it is wrong to disagree with a reviewer's assessment or an editorial decision, but when doing so it is helpful to keep certain principles in mind.

Authors who remain respectful in their disagreement will not alienate the reviewers as they would have done had they lashed out, alleging incompetence, willful delay of one's work or other conflicts of interest. Needless to say, such blanket statements have no place in any official communication with a reviewer but should be discussed, if at all, only with the editor and should be based on evidence rather than speculation. Reviewers do occasionally make factual errors, and it is important for authors to point these out, with concrete examples and without any judgment on the reviewer's general level of competence or character.

In responding to referees' comments, savvy authors address each query by each reviewer rather than cherry-picking the comments they deem relevant. They abstain from manipulative tactics, such as rephrasing a reviewer's comment in the attempt to transform it into a statement the author finds palatable. If authors do not think a point is pertinent to the work, they should give a scientific reason. In cases in which an author does not understand or disagrees with a particular criticism, it is best to say so respectfully and give one's interpretation of the results rather than merely claim that the reviewer is clueless.

It must be said that reviewers also occasionally get carried away by their dislike of a certain paper and swap professional criticism for personal attacks on a scientist's integrity. If a reviewer is worried about issues other than the scientific aspects of the paper, the best place to bring these up is in the confidential comments to editors. This will give the editors a chance to seek further clarification and determine whether, and to what extent, the concerns should affect the decision on the paper.

Please visit us at Methagora for a more practical look at how to effectively communicate with editors and reviewers. 